\title{
Review
}

\section{Dystrophin Analysis in Clinical Trials}

\author{
Annemieke Aartsma-Rus ${ }^{\mathrm{a}, \mathrm{b}, *}$ \\ ${ }^{a}$ Department of Human Genetics, Leiden University Medical Center, Leiden, The Netherlands \\ ${ }^{\mathrm{b}}$ Institute of Genetic Medicine, Newcastle University, Newcastle, UK
}

\begin{abstract}
Duchenne muscular dystrophy (DMD) is a severe muscle-wasting disease with unmet medical need. The disease is caused by mutations that disrupt the open reading frame of the dystrophin protein that is required to maintain muscle fiber stability during contraction. Lacking dystrophin patients' muscle fibers are continuously damaged eventually leading to replacement of muscle tissue by fibrotic and adipose tissues and loss of muscle function. Many therapeutic approaches aiming at dystrophin restoration are in development, and some have been or are being tested in clinical trials. For these approaches, showing dystrophin restoration or increased dystrophin expression could serve as a pharmacodynamic biomarker to confirm mechanism of action. This review provides an overview of methods currently in use to assess dystrophin in clinical trial muscle biopsies and discusses challenges of dystrophin quantification and using dystrophin as a biomarker in clinical trials.
\end{abstract}

Keywords: Dystrophin, clinical trial, Duchenne muscular dystrophy, therapy, western blotting

\section{INTRODUCTION}

The skeletal musculature makes up $\sim 30-40 \%$ of the human body, which contains over 500 different muscles. Muscles are connected to bones on both ends with tendons, and consist of post-mitotic muscle fibers that can contract in a concerted fashion to bring about movement. In most tissues integrins, transmembrane proteins involved in the connection of a cell to other cells or to the extracellular matrix, are sufficient to prevent membrane damage. However, during muscle contraction the strain on the muscle fiber membrane is beyond that experienced by cell membranes of other tissues. Therefore, the integrin connection by itself is insufficient to prevent muscle fiber damage, and a second connection is required to ensure muscle fiber integrity during contraction. This system is the dystrophin-glycoprotein associated complex, which connects the cytoskeleton actin to laminin,

${ }^{*}$ Correspondence to: Annemieke Aartsma-Rus, LUMC Postzone S4-P, Albinusdreef 2, 2333 ZA Leiden, The Netherlands. Tel.: +31 715269436; Fax: +31 715268285; E-mail: a.m.rus@lumc.nl. a constituent of the layer of connective tissue that surrounds each muscle fiber and connects muscle fibers to each other [1].

The fact that the stability provided by integrins is not enough to prevent muscle damage is underlined by the fact that patients with mutations in genes encoding dystrophin or associated proteins suffer from muscular dystrophies [2]. The most frequent and severe of these diseases is Duchenne muscular dystrophy (DMD), which is caused by mutations that disrupt the open reading frame of the dystrophin gene [3, 4]. This results in a premature truncation of translation from the dystrophin mRNA and complete loss of protein function, since the link between the actin cytoskeleton and extracellular matrix can no longer be made. As such, muscle fibers of DMD patients are very sensitive to injury during muscle contraction, resulting in a permanent state of muscle damage and chronic inflammation, which impedes muscle regeneration and instead gives rise to fibrotic and adipose tissue formation [5]. Thus, patients continuously and relentlessly loose muscle tissue and muscle function. No therapy is currently available for DMD patients, but symptomatic 


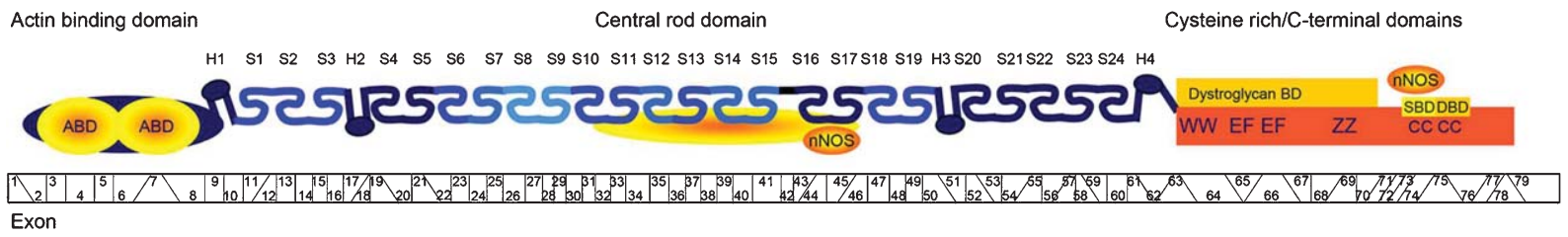

Fig. 1. Schematic depiction of the dystrophin protein (adapted from [51]). The dystrophin protein consists of $2 \mathrm{~N}$-terminal actin binding domains (ABD) encoded by exon 2-8. The central rod domain contains 24 spectrin-like repeats (S1-24) and 4 hinge regions (H1-4), a potential 5th hinge domain may exist between S15 and S16. Spectrin-like repeats each consist of 3 alpha helices that can be unfolded when force is applied to both ends. Individual spectrin-like repeats are not all equally stable (the darker the repeat the more stable it is [51]). S11-17 contain a third ABD and S16-17 contain the nNOS binding site. Furthermore, S1-3 and S4-19 have been shown to be able to interact with phospholipids [52]. The C-terminal domain contains a cystein-rich domain encoded by exon 64-70 which consists of a WW and ZZ domains involved in dystroglycan binding and 2 EF hands. The C-terminal domain contains 2 coiled coil domains (CC) involved in binding syntrophin (SBD) and dystrobrevin (DBD). Syntrophin in turn can bind nNOS. The dystrophin transcript (exons and their phasing) is shown underneath the dystrophin protein, indicating which of the domains are encoded by which exons.

Table 1

Overview of clinical trials with approaches aiming at dystrophin restoration

\begin{tabular}{|c|c|c|c|}
\hline & Phase & Trial identifier & Status \\
\hline \multicolumn{4}{|l|}{ A. Cell therapy } \\
\hline \multicolumn{4}{|l|}{ Cell type } \\
\hline Myoblast & 1 & None found & Published [26] \\
\hline Mesangioblast & 1 & None found & Ongoing \\
\hline \multicolumn{4}{|l|}{ B. Exon skipping } \\
\hline \multicolumn{4}{|l|}{ Compound } \\
\hline \multirow[t]{6}{*}{ Drisapersen } & 1 (intramuscular) & NTR $1241^{1}$ & Published [22] \\
\hline & 1 (single dose in nonambulant patients) & NCT01128855² & Published [53] \\
\hline & $2 \mathrm{a}($ dose escalation $)$ & NCT01910649 & Published [21] \\
\hline & 2 (dose regimen) & NCT01153932 & Complete \\
\hline & 2 (dose comparison) & NCT01462292 & Complete \\
\hline & 3 & NCT01254019 & Complete \\
\hline \multirow[t]{3}{*}{ Eteplirsen } & 1 & NCT00159250 & Published [20] \\
\hline & $2 \mathrm{a}$ & NCT00844597 & Published [19] \\
\hline & $2 b$ & NCT01396239 & Published [18] \\
\hline PRO044 & $2 \mathrm{a}$ & NCT01037309 & Complete \\
\hline PRO045 & $2 \mathrm{a}$ & NCT01826474 & Ongoing \\
\hline PRO053 & $2 \mathrm{a}$ & NCT01957059 & Ongoing \\
\hline \multicolumn{4}{|l|}{ C. Gene therapy } \\
\hline \multicolumn{4}{|l|}{ Vector } \\
\hline rAAV2.5-CMV-minidystrophin & 1 & NCT00428935 & Published [24] \\
\hline \multicolumn{4}{|l|}{ D. Stop codon readthrough } \\
\hline \multicolumn{4}{|l|}{ Compound } \\
\hline \multirow[t]{4}{*}{ Ataluren } & 1 & None found & Published [54] \\
\hline & $2 \mathrm{a}$ & NCT00264888 & Published [17] \\
\hline & $2 b$ & NCT00592553 & Complete \\
\hline & 3 & NCT01826487 & Ongoing \\
\hline Gentamicin & 2 & NCT00451074 & Published [16] \\
\hline
\end{tabular}

${ }^{1}$ Netherlands trial registry. ${ }^{2}$ Clinicaltrials.gov registry.

treatment and care is offered to patients in the Western world that can slow down the disease to some extent $[6,7]$. Nevertheless, most patients become wheelchairdependent in their teens, require assisted ventilation in the late teens or early twenties and die due to respiratory or cardiac complications in the second to fourth decade.

In addition to DMD, mutations in the dystrophin gene can also give rise to Becker muscular dystrophy (BMD) [3]. Notably, here mutations maintain the open reading frame, allowing the production of an internally deleted protein, which maintains its crucial domains for binding to actin and the dystrophin associated complex. The severity of BMD varies, with some patients being almost as severe as DMD but also patients being diagnosed in the fifth or sixth decade of life. Generally, BMD patients have a less progressive disease than DMD patients, remain ambulant for ten to fifteen year after diagnosis, do not need assisted ventilation and have near normal life expectancy [2]. 
Dystrophin (Fig. 1) contains 2 N-terminal actinbinding domains, a central rod domain consisting of 24 spectrin-like repeats and 4 hinge regions, a cysteinerich domain and a C-terminal domain [8]. The central rod domain contains a third actin-binding domain that is located in repeats 11-17 and a binding site for neuronal nitric oxide synthase (nNOS) located in repeats 16 and 17 [9]. The cysteine-rich domain contains YY and WW domains involved in binding to betadystroglycan and EF hand domains. The C-terminal domain binds to syntrophin, which in turn can also bind to nNOS, and a domain that binds to dystrobrevin $[1,8]$.

The dystrophin gene is huge (a stunning $2.2 \mathrm{Mb}$, making up $\sim 10 \%$ of the $\mathrm{X}$-chromosome and close to $0.1 \%$ of the human genome). While the mRNA is larger $(14 \mathrm{~kb})$ than the average transcript, the 79 exons only contribute to $0.7 \%$ of the gene, with excessively long introns accounting for the other $99.3 \%$. The majority of DMD and BMD patients carry deletions of one or more exons. The size and location of in-frame deletions have taught the field a lot about the relative importance of dystrophin domains depicted in Fig. 1 (reviewed in [10]). Mutations that abolish all three actin-binding domains or that affect the cysteine rich domain invariably result in DMD regardless of whether mutations are in-, or out-of-frame, and even missense mutations in the dystroglycan-binding domain have been found to cause DMD [11]. As there are three actin-binding sites, there is some redundancy here and in-frame mutations that leave at least one actin-binding site intact generally lead to BMD, although patients with only a single actin binding site often have a relatively severe disease progression. Most BMD patients have mutations that delete part of the central rod domain and provided that at least one third of this domain is maintained these deletions do not abolish dystrophin function. The nNOS binding domain encoded by exon $42-45$ is a relatively recent discovery (until then, it was thought nNOS recruitment through the dystrophinglycoprotein associated complex was mediated only through syntrophin) [9]. The nNOS protein plays a role in functional sympatholysis (ensuring contracting muscle receives enough blood). The nNOS enzyme can generate $\mathrm{NO}$, which leads to blood vessel dilation [12]. When NO production fails because nNOS is not located near the muscle membrane, as is the case for DMD patients and a subset of BMD patients, there is a risk for ischemia during exercise, which can obviously exacerbate the muscle damage already occurring in DMD and BMD patients [13]. However, the contribution of the presence or absence of the nNOS binding domain encoded by exon $42-45$ to disease severity is not yet fully elucidated and studying this is hampered by the fact that indirect nNOS recruitment is also possible through syntrophin.

What is clear is that without dystrophin, DMD patients suffer from a very severe, debilitating disease, characterized by loss of one function after another. Towards developing a therapy for DMD patients, different approaches to restore dystrophin are in development, such as stop codon read through, exon skipping, gene therapy and cell therapy $[14,15]$. Stop codon read through can be achieved by e.g. gentamicin and ataluren and applies to patients carrying a premature stop codon ( $\sim 13 \%$ of DMD patients) $[16,17]$. The exon skipping approach aims to reframe dystrophin transcripts by skipping an exon from the pre-mRNA to allow production of BMD-like dystrophins using antisense oligonucleotides (AONs) that specifically bind to a target exon [18-22]. Different chemically modified AONs are currently evaluated in clinical trials, the $2^{\prime}$ - $O$-methyl phosphorothioate (drisapersen, PRO044, PRO045 and PRO053 targeting exons 51, 44, 45 and 53) and the phosphorodiamidate morpholino oligomer (eteplirsen targeting exon 51) [23]. For gene therapy generally artificially constructed mini-dystrophins are used, since the coding sequence of the full length dystrophin is larger than the capacity of the adeno-associated viral vector the only virus which can transduce muscle with good efficiency [24]. These mini-dystrophins contain only the most crucial domains (the first two actin-binding domains, 4 spectrin repeat domains, 2-3 hinge domains and the cysteine rich domains) and have been shown to slow down disease progression in animal models [25]. Cell therapy involves either transplanting cells with myogenic potential from a healthy donor, or autologous transplantation where patient-derived cells are genetically modified with gene therapy ex vivo $[14$, 26]. These therapeutic approaches have been and are being tested clinical trials in DMD patients (Table 1). For market approval by e.g. the European Medicine Agency (EMA) and US Food and Drug Administration (FDA) therapies have to show clinical benefit [27]. However, as these therapeutic approaches all aim at restoration of dystrophin, showing dystrophin restoration or increased expression in a muscle biopsy would serve as an obvious pharmacodynamic biomarker to confirm mechanism of action. Unfortunately, dystrophin analysis and quantification are challenging due to e.g. the low abundance of the protein, its size and the fact that many DMD patients have a low level of dystrophin positive 'revertant fibers' [28]. This review 
will provide an overview of different methods for dystrophin analysis that have been reported so far for clinical trials and will discuss how dystrophin analysis can guide clinical trials and therapy development.

\section{METHODS TO DETECT AND QUANTIFY DYSTROPHIN IN CLINICAL TRIALS}

Dystrophin is a challenging protein to detect and quantify, due to its size $(427 \mathrm{kDa})$, low abundance ( $0.002 \%$ of total muscle protein) and the presence of revertant fibers. Numerous methods have been reported to detect and/or quantify dystrophin in a diagnostic or research setting, such as immunohistochemical or fluorescence analysis [29, 30], western blotting [31], enzyme linked immuno sorbant assay (ELISA) [32], mass spectrometry [33] and a reverse protein assay [34]. It is good to bear in mind that western blotting and immunohistochemistry/fluorescence are semiquantitative methods, while ELISA and mass spectrometry based methods potentially allow absolute quantification. However, this requires a characterised standard of known concentration and linearity of signal. Unfortunately, dystrophin protein of known concentration has not been used so far, and therefore these methods are for now quasi or semi quantitative at best. Furthermore, except one report, no one has been able to get an ELISA-based system operational for dystrophin quantification despite extensive efforts (my own unpublished observation (2012), and unpublished observations from EP Hoffman, Children's National Medical Center, Washington DC, US (2013); V Arechavala-Gomeza, BioCruces Health Research Institute, Barakaldo, Spain (2012) and JCT van Deutekom, Prosensa Therapeutics, Leiden, the Netherlands (2009)). In our hands the ELISA system was suboptimal, because the signal for healthy muscle was similar to, or only slightly above the background signals. These high backgrounds occurred for all dystrophin antibodies tested and with various blocking methods, suggesting that perhaps (most) dystrophin antibodies have a high affinity for plastics. Mass spectrometry for dystrophin quantification, using total human healthy muscle protein extract containing dystrophin as a 'standard', is a newly developed method that has so far not been used to quantify clinical trial samples and therefore will not be discussed in this review. Instead it will focus on the two methods that have been used so far to detect dystrophin in biopsy samples: western blotting and immunofluorescence analyses.
For western blotting samples (in this case muscle biopsies) are lysed and the protein lysate is loaded on a denaturing polyacrylamide gel to separate for size [31]. The gel is then blotted onto a membrane and antibodies are used to detect the protein of interest (dystrophin in this case). Secondary antibodies containing conjugates (fluorescent or infrared dyes, or enzymes able to react with chemiluminescence substrates) are used to visualize the target protein. Generally a concentration series of healthy muscle protein lysate is taken along, while co-staining with an antibody for a reference protein is used to ensure equal loading of samples. This allows semi-quantification, where the intensity or density of the dystrophin band is a measure for quantity, while the height of the band on the blot is a measure for its size.

For immunofluorescence analysis muscle crosssections are incubated with a dystrophin specific primary antibody, followed by incubation with secondary antibodies carrying a fluorescent conjugate specific for the species in which the primary antibody was raised in. Often co-staining with muscle specific proteins is used (e.g. laminin or spectrin) to allow identification of muscle fibers and/or to have an internal reference [29, 30]. Cross-sections of healthy muscle can be used as an external reference, while cross-sections incubated with only the primary and only the secondary antibodies can be used for background correction. Immunofluorescence analysis does not provide information on protein size, however, unlike western blotting it does reveal where the dystrophin is located and how it is distributed over muscle fibers (e.g. 10\% of muscle fibers with $100 \%$ dystrophin and $100 \%$ of muscle fibers with $10 \%$ of dystrophin will both lead to $\sim 10 \%$ of dystrophin by western blotting) and whether the dystrophin can interact with associated proteins [35]. When a muscle specific reference protein is taken along, it also provides information on muscle quality (the number of muscle fibers in the biopsy). In immunofluorescence analysis assays, dilutions are not possible and hence linearity of the response cannot be demonstrated. Dystrophin "quantification" can be done in several ways (see below) but it is always relative and never absolute.

As neither method provides a complete picture, both techniques are often used in combination for biopsies taken in trials testing therapeutic approaches aiming at dystrophin restoration. Table 2 contains details on methods used for dystrophin analysis that have been published or submitted for clinical trial sample analysis [16-22, 24, 26] (and unpublished observations A Lourbakos, Prosensa Therapeutics, Leiden, the 


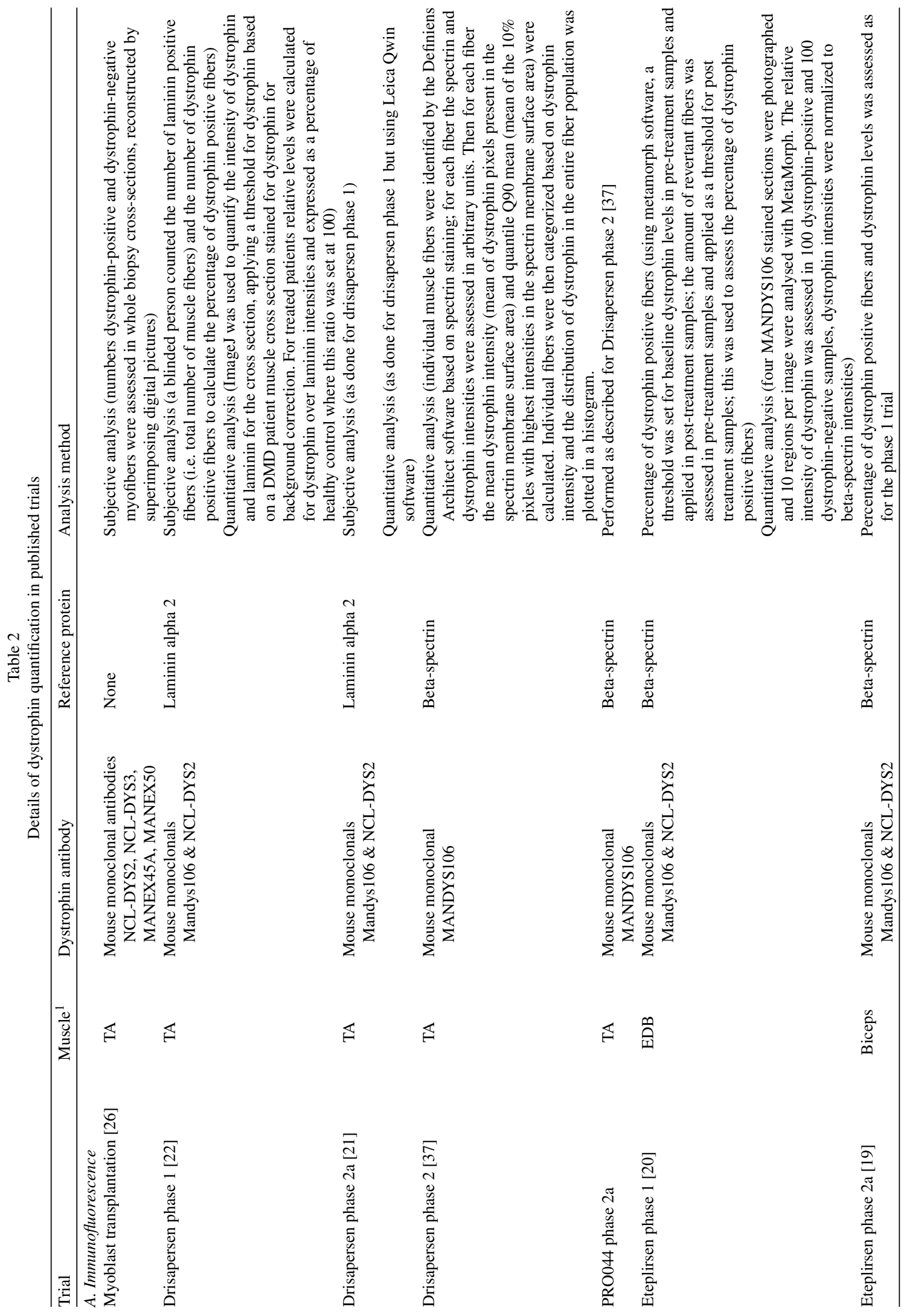




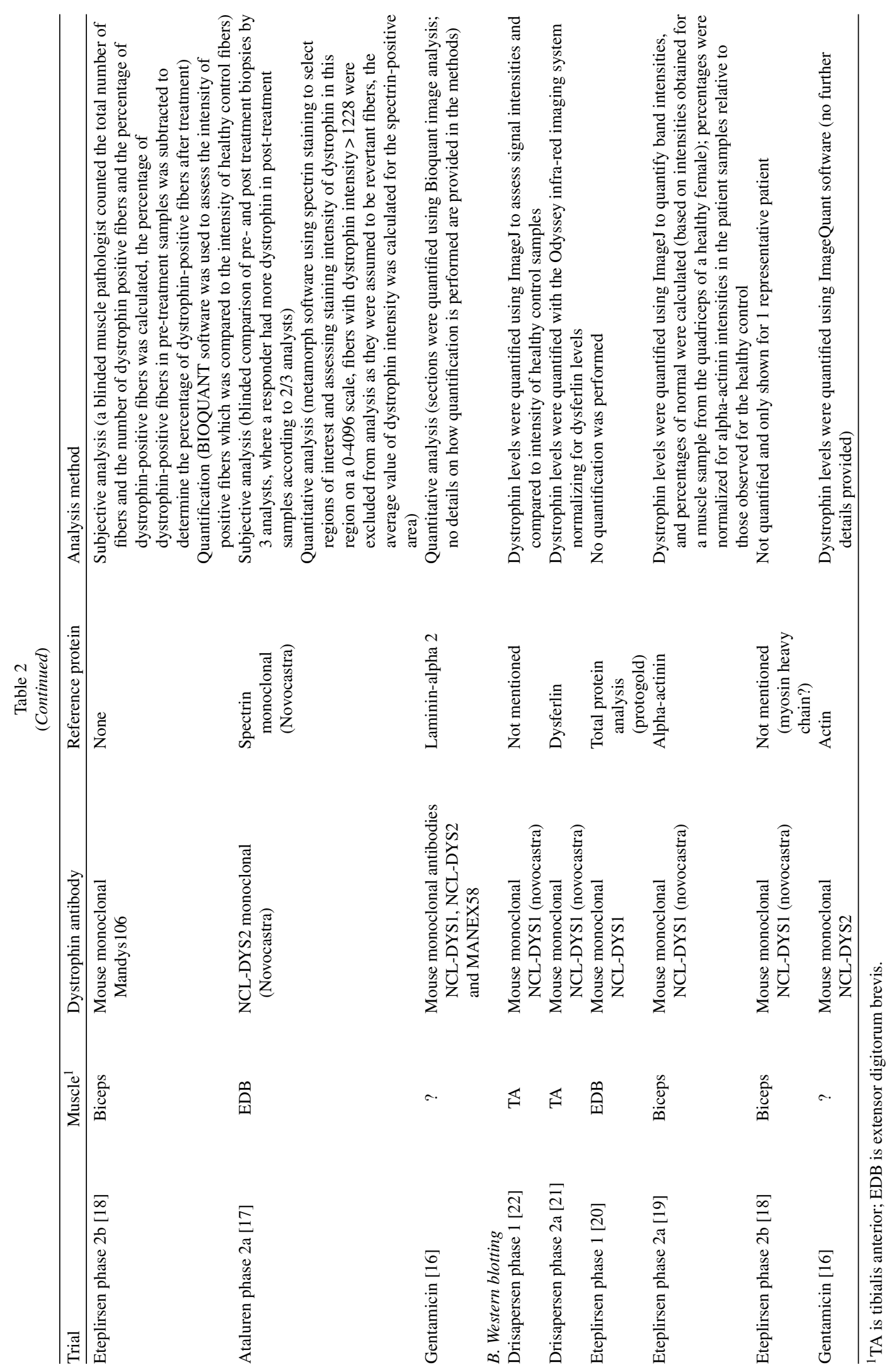


Netherlands (2013)). Notably, for all published trials immunofluorescence analysis was performed, while western blotting was performed for only a subset of trials and not always for all patients. The most likely reason for this is that immunofluorescence is more sensitive than Western blotting and also that less sample is required for immunofluorescence analysis. As can be appreciated from Table 2 for both western blotting and immunofluorescence analyses different protocols are in use. The western blotting protocol used is based on an original protocol published by Anderson et al. [31], although each group has introduced adaptations and uses e.g. different loading controls, types of polyacrylamide gels and gel running and blotting protocols. For immunofluorescence standardized protocols have been published by two different groups and submitted by a third group. The first method was published by Arechavala-Gomeza et al and has been developed in the group of Francesco Muntoni ("Muntoni method") [29] and has been used to analyse muscle biopsies from phase 1 and $2 \mathrm{a}$ eteplirsen exon skipping trials $[19,20]$. The second method was published by Taylor et al in 2012 and has been developed by the group of Kevin Flanigan ("Flanigan method") [30] and was used to analyse muscle biopsies from the phase 2a ataluren trial [17]. The Muntoni method uses four images from different areas of the muscle cross section selected at random while out of focus and then captured in focus. Ten regions are selected from each image (so per cross section 40 measurements are taken), with the proviso that when regions are selected in the center of a fiber, an area of fibrosis, on a neuromuscular junction, or in a previously selected fiber, the region is moved to the nearest fiber membrane. Each region contains both a portion of sarcolemma and cytoplasm. Then for each region the intensity value of the fluorescence is assessed by MetaMorph in a dynamic range of 0-4095 intensity units. Since 4095 is the maximum that can be measured, it is crucial to prevent saturation (i.e. making sure all intensities are well below 4095, also for the control muscle). To correct for background the minimal intensity (i.e. the value of the cytoplasm) is subtracted from the maximum intensity. Then each of the 40 values are normalized with the beta-spectrin intensity measurements measured from different cross section of the same biopsy and plotted and presented as a scatter plot and summarized as a percentage ratio of control. Using this method is was possible to distinguish dystrophin levels in samples from DMD and BMD patients and controls, where very low levels were observed for DMD patients, and intermediate levels (between control and DMD) were observed for BMD patients [29].

The Flanigan method also uses a beta-spectrin dystrophin co-staining, but selects fields while viewing the spectrin signal under 20 times magnification. Image acquisition parameters (e.g. pinhole size, exposure time) are set for dystrophin and spectrin channels with a normal control tissue and then subsequently used to capture all samples. For each sample four nonoverlapping images are acquired and analyzed with MetaMorph. First a signal threshold for spectrin intensity is set manually. All signals over the threshold make up the spectrin region of interest, which is converted into a binary mask. In a subsequent step all spectrin areas smaller than 3 by 3 contiguous pixels are removed to obtain a contiguous muscle membrane. This binary image is then dilated to create a spectrin mask that is overlaid on the spectrin and dystrophin images and the intensities are quantified for both. This method thus obtains dystrophin intensities for the sarcolemma (stained by spectrin) of the entire image. The sum of the dystrophin intensities and sum of spectrin intensities are calculated, and the dystrophin to spectrin ratio is calculated and defined as the average dystrophin intensity for the image. The total spectrin intensity value is divided by the total area of spectrin-positive regions, resulting in the average spectrin intensity for the whole image. Finally, by dividing the average dystrophin intensity by the average spectrin intensity the dystrophin/spectrin ratio is obtained. The methods paper showed that spectrin intensity is similar for DMD, BMD and control samples and also is similar between individual samples from DMD and BMD patients (thus validating spectrin as a good reference protein for DMD and BMD immunofluorescence analysis) [30]. Finally, a good correlation between average dystrophin intensities relative to control samples as assessed by immunofluorescence and dystrophin levels as assessed by Western blotting were observed. For analysis of clinical trials samples, revertant fibers can be excluded in pre- and post-treatment samples by applying a maximum threshold.

A third method is currently in use by Prosensa Therapeutics ("Lourbakos method") and was presented at the World Muscle Society meeting 2013 [36] and has been submitted for publication (unpublished observations, C Beekman and A Lourbakos, Prosensa Therapeutics, Leiden, the Netherlands (2013)). Biopsy quality was assessed by hematoxylin and eosin staining, which was also used to evaluate freezing artefacts and muscle quality (relative amount of fibrotic and adipose tissue). Per biopsy, 2-4 sections are stained with 
dystrophin and spectrin antibodies, and per section 5 non overlapping images are acquired by confocal microscopy at 25 times magnification. Areas containing very few muscle fibers or extensive fibrosis or adiposis were avoided. Parameters such as pinhole size, exposure time etc were standardized, for studies comparing healthy, BMD and DMD biopsies these were set based on healthy muscle samples; for studies comparing DMD biopsies pre- and post treatment, fluorescent beads were used to set imaging parameters. The Definiens Architect image analysis software version 2.0 identifies all individual muscle fibers in one image, and identifies for each fiber the membrane and the cytoplasm based on the spectrin signal. For each fiber dystrophin and spectrin intensities are measured in arbitrary units. Then, for each fiber the mean dystrophin intensity is calculated (i.e. the mean of all dystrophin pixels present in the membrane surface area) as well as the mean of the $10 \%$ pixels with the highest intensity in the spectrin membrane surface area (the Q90-mean). Revertant fibers are readily detected (because of their high dystrophin intensity) and excluded from further analysis. Individual fibers are then 'binned' based on their dystrophin intensity and the distribution of dystrophin intensity of the entire fiber population is then plotted as a histogram of the percentage of fibers versus dystrophin intensity. The method was shown to be reproducible between experiments and between replicate samples and operators and a good correlation was found between different dystrophin antibodies.

Using this analysis, Beekman and colleagues showed that dystrophin levels vary between fibers in healthy muscle (up to threefold) as had been reported before $[19,29,30]$. They also observed variation in dystrophin levels between fibers from different muscles from one individual (lowest in tibialis anterior and highest in gastrocnemius, 30\% difference)(unpublished observations, C Beekman and A Lourbakos, Prosensa Therapeutics, Leiden, the Netherlands (2013)). Furthermore, (very) low levels of dystrophin ("trace amounts") can be detected for most DMD patients, while intermediate levels were found for BMD patients [36]. Like for healthy muscle, dystrophin intensities varied between fibers for BMD and DMD patients (up to three-fold) (unpublished observations, C Beekman and A Lourbakos, Prosensa Therapeutics, Leiden, the Netherlands (2013)). Notably, after treatment of patients in the phase 2 dose regimen study with drisapersen, a shift towards higher dystrophin intensities could be observed in the majority of post-treatment samples compared to pre- treatment fibers [37]. Furthermore, a 30\% increase in intensities was observed for post-treatment samples vs pre-treatment samples from patients involved in the phase $2 \mathrm{a}$ trial with PRO044 for exon 44 skipping [36](unpublished observations, C Beekman and A Lourbakos, Prosensa Therapeutics, Leiden, the Netherlands (2013)). The method also revealed higher intensities for spectrin in DMD and BMD samples than healthy samples (unpublished observations, C Beekman and A Lourbakos, Prosensa Therapeutics, Leiden, the Netherlands (2013)). However, since the dystrophin quantification does not rely on spectrin intensities (spectrin staining is only used to generate the mask), this will not influence dystrophin quantification for this method.

Comparison between groups can only be done when the same reference proteins are used. For the immunofluorescence method there now appears to be a consensus that beta-spectrin is the best choice. This reference is used for each of the most recent trials (Table 2) and it has also been confirmed that betaspectrin levels in fibers are reasonably stable between individuals, and between DMD, BMD and healthy individuals [30], although the latter was not found by Beekman and colleagues (unpublished observations, C Beekman and A Lourbakos, Prosensa Therapeutics, Leiden, the Netherlands (2013)). For western blotting there is more variation in the choice of reference protein/loading control. Because dystrophin is such a large protein, low percentage or gradient polyacrylamide gels are generally run overnight and the commonly used loading controls (small proteins such as betaactin and alpha-tubulin) will no longer be present in the gel. So ideally a larger protein is used as a reference. Myosin heavy chain ( $200 \mathrm{kDa}$ ) has been used for western blots on cell lysates of cultured cells [38]. However, due to its extremely high abundance in skeletal muscle ( $\sim 45 \%$ of total muscle) this is not a good loading control, since it oversaturation is impossible to prevent. Of the various reference proteins used, alpha-actinin is probably the most attractive, since expression is similar between healthy and dystrophic muscle at least in mice (my own unpublished observations (2013)). However, since alpha-actinin is a higher abundance protein, lack of saturation of the alpha-actinin signal needs to be demonstrated, because oversaturated signals cannot be used for normalisation. Alpha-actinin also allows correction for muscle quality, since it is not expressed by fibrotic and adipose tissues. Spectrin, a lower abundance protein in muscle for which the signal saturates at higher amounts of protein input, has also been used by Prosensa. 


\section{STANDARDIZATION OF DYSTROPHIN QUANTIFICATION IN CLINICAL TRIALS}

The question is of course how well the different methods that are used by different groups compare (and more importantly how comparable the dystrophin levels reported in various trials are). This issue was also raised in a workshop hosted by the EMA in 2009 on the development of exon skipping therapies for DMD [39], and resulted in an effort coordinated by Francesco Muntoni to compare the Flanigan and Muntoni methods. This endeavour involved different expert laboratories (Muntoni (University College London, UK), Flanigan (Nationwide Children's Hospital, Columbus OH, USA), Prosensa Therapeutics (Leiden, the Netherlands), Voit (Institute de Myologie, Paris, France) and Straub (Newcastle University, UK). Muscle biopsy samples from DMD and BMD patients were divided in 5 pieces and distributed to the 5 centres in a blinded fashion, along with 2 samples from healthy controls. Each centre performed the quantification with the two methods; Prosensa also used the Lourbakos method. Notably, the dystrophin levels for the different patient samples concurred very well between laboratories for each of the methods, and also for the two different methods within laboratories (or three methods for Prosensa). Furthermore, the results correlated with quantification performed by western blotting (unpublished observation K Anthony and F Muntoni, University College London, UK (2014)). This suggests that, at least when using the same control samples and performing the analyses in a standardized setting, these methods are comparable.

When assessing dystrophin levels in a clinical trial setting, the protocol should of course be standardized as much as possible. Differences in obtaining, handling, storing and shipping muscle biopsy samples can account for a lot of variation between biopsy samples, especially for multicenter trials [40]. Ideally, standardized operating procedures should be in place and people obtaining, handling and receiving the biopsy should be trained and experienced couriers should be used for shipping. Sending duplicate biopsies at two different time points is a way to avoid precious samples being lost during shipping.

The quantification of dystrophin for clinical trials is challenging and laborious and poses a number of specific challenges. In the past it was always assumed that muscle fibers from DMD patients did not produce dystrophin, aside from the occasional revertant fibers. However, with more sophisticated microscopes, cameras and detectors it has now become apparent that almost all fibers from DMD patients express trace amounts of dystrophin [28, 36] and [29](unpublished observation C Beekman and A Lourbakos, Prosensa Therapeutics, Leiden, the Netherlands (2013)). The levels of these trace amounts vary between fibers and between patients and generally patients will also have revertant fibers where dystrophin is expressed at high levels. This 'pattern' can only be detected with immunofluorescence, and the analysis is not a matter of showing dystrophin restoration after treatment where before treatment there was none, but of showing higher levels of dystrophin after treatment than before. Thus, the analysis should be both sensitive and accurate and both pre- and post-treatment biopsies are required. Analysis is further hampered by the fact that dystrophin levels even vary between different fibers in a cross-section for healthy muscles and that different dystrophin levels are expressed in different muscles in mice [41] and man (unpublished observations, C Beekman and A Lourbakos, Prosensa Therapeutics, Leiden, the Netherlands (2013)). This underlines that it is important to use a control sample derived from the same muscle type as the clinical trial biopsies to prevent over- or underestimation of wild type levels for that muscle. Since dystrophin levels vary also between individuals, ideally different groups should use the same reference sample. In fact, one of the reasons the three different methods concurred as well as they did, may be that all groups used the same reference control samples. Obviously, control samples generally are in short supply, so having a common pool of human control muscle is probably not realistic. A transgenic animal model expressing human dystrophin in a mouse dystrophin-negative background is available and muscles harvested from this animal may provide a more accessible common source for control muscle [42]. However, this is mouse tissue and generally mouse monoclonal antibodies are used for Western blotting and immunofluorescence. It will have to be established whether there is interference with secondary antibodies recognizing mouse $\mathrm{IgGs}$ in muscle.

A further complication of dystrophin assessment in clinical trial samples is the muscle quality of the biopsy. Due to the lack of dystrophin, muscle fibers will be replaced by fibrotic and adipose tissues in DMD patients and muscle quality of patients will deteriorate over time. However, the level and rate of deterioration is not similar for each muscle (e.g. the quadriceps is generally affected in early stages of the disease, while the tibialis anterior and posterior are reasonably well preserved initially [43]). Furthermore, muscle quality varies within individual muscles, where often patches 
of fibrosis and adipose tissue can be found. Thus, the quality of a muscle biopsy is not necessarily representative for the quality of the whole musculature of the patient. Finally, since dystrophin is not expressed by fibrotic and adipose tissue but only by muscle tissue, the dystrophin levels observed in a muscle biopsy do not necessarily reflect the dystrophin levels in the whole musculature of the patient. Magnetic resonance imaging (MRI) provides a more general picture of muscle quality in a limb and, provided that MRI is available in the trial site, MRI can be used to guide obtaining a biopsy from a reasonably well preserved area.

\section{TOWARDS DEVELOPING DYSTROPHIN AS A BIOMARKER}

Given the invasiveness of muscle biopsies, especially for patients who have a limited amount of muscle tissue to begin with, one could question whether muscle biopsies should be performed for clinical trials aiming at dystrophin restoration. Market approval of treatments by regulators will not be based on the presence of dystrophin but on "clinical benefit", i.e. functional improvement of DMD patients compared to the natural history of the disease. For DMD the most commonly used primary outcome measure in current trials (i.e. the outcome measure measuring functional benefit) is the 6 minute walk test, where a significant difference of $\sim 30$ meters between treated and placebo groups has been proposed to reflect clinical benefit [44].

Since dystrophin prevents muscle fiber damage, restoring dystrophin in DMD patients will hopefully slow down further disease progression. It will, however, most likely not restore muscle tissue that has been lost. A slower disease progression qualifies as a valid clinically important outcome for regulatory agencies, but it is obviously more challenging to detect a significant difference when looking for a slower decline muscle function than for improved muscle function, especially in a disease with a relatively slow disease progression (a decline of $\sim 30$ meters per year for most ambulant patients) [45-47]. As such, dystrophin restoration is a valuable pharmacodynamic biomarker to confirm mechanism of action of the treatment and could provide additional proof for a treatment effect.

It has been proposed to use dystrophin as a surrogate endpoint for clinical trials aiming at dystrophin restoration. A surrogate endpoint is an outcome that is used instead of the primary outcome measure. As such the surrogate endpoint has to correlate to, or be predictive of clinical benefit [27]. DMD is caused by lack of dystrophin and patients with low levels (>20\%) of partially functional dystrophin (BMD patients) have a significantly different disease progression [48]. Furthermore, recent studies in animal models suggest that lower levels of dystrophin $(<4 \%)$ are sufficient to significantly prolong survival in a dystrophin-utrophin negative background $[49,50]$. Also in humans, very low levels of dystrophin can slow down disease progression, as underlined by the finding that patients with a deletion flanking exon 44 have a significantly slower disease course than other deletion patients [45]. This is most likely the result of spontaneous low level exon 44 skipping, and the production of very low levels of dystrophin in this subset of patients. Indeed, it has been reported that trace dystrophin levels in pre-treatment samples of patients involved in an exon 44 skipping trial are higher than in pre-treatment of patients involved in exon 51 skipping trials [36] (unpublished observations C Beekman and A Lourbakos, Prosensa Therapeutics, Leiden, the Netherlands (2013)). Thus, arguably, some dystrophin is better than none and as such restoring dystrophin in DMD patients would be expected to lead to clinical benefit and dystrophin should be an acceptable surrogate endpoint. Unfortunately it is not as simple as that. First, in the examples described here, patients have low levels of dystrophin from birth. By contrast, in DMD patients involved in exon skipping trials, dystrophin is restored at a later time point, when muscle quality will already be affected by the disease. As dystrophin is needed to prevent muscle damage, it is anticipated that restoring dystrophin in these muscles will slow down further muscle damage. The amount of functional benefit that can be expected for a given patient will depend on both the level of dystrophin produced in the muscle and the muscle quality. However, it is not yet known which levels of dystrophin are needed to slow down disease progression, whether the amount of dystrophin needed to achieve this, is the same for early and late stage patients, and whether dystrophin restoration is unable to lead to functional benefit when a certain amount of muscle damage has accumulated. Due to these unknowns dystrophin is as yet not a surrogate endpoint for DMD. Another issue is that surrogate endpoint validation involves showing accuracy and reproducibility of the assay, all of which could prove difficult for dystrophin quantification due to the challenges pointed out throughout the manuscript.

For the exon skipping approach, the matter is further complicated by the fact that different internally deleted dystrophins can be generated through skipping 
of a single exon (e.g. exon 51 skipping can restore the reading frame for a deletion of exon 47-50, 48-50, 49-50, 50 and 52). The stability and functionality of the different dystrophins will likely vary, presenting yet another parameter that can influence the extent of functional benefit any given patient may have from the exon skipping approach.

Most of the therapeutic approaches currently in development for DMD are personalized medicine type of approaches and will apply to only subsets of patients. This will make showing clinical benefit with the 6 minute walk test challenging, since most likely not all patients with eligible mutations will be ambulant and for those who are there will be variation in baseline performance. Therefore, even though dystrophin restoration is not an acceptable surrogate endpoint at this point in time, hopefully it will be an acceptable endpoint for e.g. exceptional, conditional or accelerated approval mechanisms once clinical benefit has been shown for one or a few dystrophin restoring approaches. Towards this, Academics and Industry should continue working towards standardized protocols and common references both for preclinical and clinical work. However, this effort should also involve regulators, to ensure that the assay adheres to criteria outlined by e.g. the FDA and EMA for biomarker qualification. Having dystrophin as a qualified biomarker would enable early market approval, pending the gathering of additional data. Since there is a clear unmet medical need for DMD, any way to accelerate access to therapies is sorely needed.

\section{ACKNOWLEDGMENTS}

Pavel Balabanov and Afrodite Lourbakos are gratefully acknowledged for useful discussions and sharing unpublished information on the Lourbakos dystrophin quantification method, respectively. The biochemical outcome measures working group of COST Action BM1207, led by Francesco Muntoni is acknowledged for sharing results of the analysis comparison.

The author receives funding from the European Union (FP7 Neuromics project no 2012-305121), the Dutch Organisation for Scientific Research (ZonMw), the Duchenne Parent Project (the Netherlands), the Prinses Beatrix Spierfonds and Spieren for Spieren, and is chair of COST Action BM1207 (www.exonskipping.eu), which supports networking to standardize dystrophin quantification methods and discussion on regulatory models for exon skipping therapies for rare diseases.

\section{DISCLOSURES}

The author is employed by LUMC, which has patent applications on exon skipping, some of which have been licensed to Prosensa Therapeutics. As co-inventor of a number of these patents, the author is entitled to a share of royalties or license fees.

\section{REFERENCES}

[1] Blake, D. J., Weir, A., Newey, S. E., and Davies, K. E. Function and genetics of dystrophin and dystrophin-related proteins in muscle. Physiol Rev. 2002; 82(2): 291-329.

[2] Emery, A. E. The muscular dystrophies. Lancet. 2002; 359(9307): 687-695.

[3] Monaco, A. P., Bertelson, C. J., Liechti-Gallati, S., Moser, H., and Kunkel, L. M. An explanation for the phenotypic differences between patients bearing partial deletions of the DMD locus. Genomics. 1988; 2(1): 90-95.

[4] Hoffman, E. P., Brown, R. H., Jr., Kunkel, L. M. Dystrophin: The protein product of the Duchenne muscular dystrophy locus. Cell 1987 Dec 24;51(6): 919-28.

[5] Grounds, M. D. Two-tiered hypotheses for Duchenne muscular dystrophy. Cell Mol Life Sci. 2008; 65(11): 1621-1625.

[6] Bushby, K., Finkel, R., Birnkrant, D. J., Case, L. E., Clemens, P. R., and Cripe, L., et al. Diagnosis and management of Duchenne muscular dystrophy, part 1: Diagnosis, and pharmacological and psychosocial management. Lancet Neurol. 2010; 9(1): 77-93.

[7] Bushby, K., Finkel, R., Birnkrant, D. J., Case, L. E., Clemens, P. R., and Cripe, L., et al. Diagnosis and management of Duchenne muscular dystrophy, part 2: Implementation of multidisciplinary care. Lancet Neurol. 2010; 9(2): 177-189.

[8] Koenig, M., Monaco, A. P., and Kunkel, L. M. The complete sequence of dystrophin predicts a rod-shaped cytoskeletal protein. Cell. 1988; 53(2): 219-228.

[9] Lai, Y., Thomas, G. D., Yue, Y., Yang, H. T., Li, D., and Long, C., et al. Dystrophins carrying spectrin-like repeats 16 and 17 anchor nNOS to the sarcolemma and enhance exercise performance in a mouse model of muscular dystrophy. J Clin Invest. 2009; 119(3): 624-635.

[10] Aartsma-Rus, A., Van Deutekom, J. C., Fokkema, I. F., Van Ommen, G. J., and Den Dunnen, J. T. Entries in the Leiden Duchenne muscular dystrophy mutation database: An overview of mutation types and paradoxical cases that confirm the reading-frame rule. Muscle Nerve. 2006; 34(2): 135-144.

[11] Vulin, A., Wein, N., Strandjord, D. M., Johnson, E. K., Findlay, A. R., and Maiti, B., et al. The ZZ domain of dystrophin in DMD: Making sense of missense mutations. Hum Mutat. 2014; 35(2): 257-264.

[12] Thomas, G. D. Functional muscle ischemia in Duchenne and Becker muscular dystrophy. Front Physiol. 2013; 4: 381.

[13] Martin, E. A., Barresi, R., Byrne, B. J., Tsimerinov, E. I., Scott, B. L., and Walker, A. E., et al. Tadalafil alleviates muscle ischemia in patients with Becker muscular dystrophy. Sci Transl Med. 2012; 4(162): 162ra155.

[14] Pichavant, C., Aartsma-Rus, A., Clemens, P. R., Davies, K. E., Dickson, G., and Takeda, S., et al. Current status of pharmaceutical and genetic therapeutic approaches to treat DMD. Mol Ther. 2011; 19(5): 830-840.

[15] Palmieri, B., Tremblay, J. P., and Daniele, L. Past, present and future of myoblast transplantation in the treatment of 
Duchenne muscular dystrophy. Pediatr Transplant. 2010; 14(7): 813-819.

[16] Malik, V., Rodino-Klapac, L. R., Viollet, L., Wall, C., King, W., and Al-Dahhak, R., et al. Gentamicin-induced readthrough of stop codons in Duchenne muscular dystrophy. Ann Neurol. 2010; 67(6): 771-780.

[17] Finkel, R. S., Flanigan, K. M., Wong, B., Bonnemann, C., Sampson, J., and Sweeney, H. L., et al. Phase 2a study of ataluren-mediated dystrophin production in patients with nonsense mutation Duchenne muscular dystrophy. PLoS One. 2013; 8(12): e81302.

[18] Mendell, J. R., Rodino-Klapac, L. R., Sahenk, Z., Roush, K., Bird, L., and Lowes, L. P., et al. Eteplirsen for the treatment of Duchenne muscular dystrophy. Ann Neurol. 2013; 74(5): 637-647.

[19] Cirak, S., Arechavala-Gomeza, V., Guglieri, M., Feng, L., Torelli, S., and Anthony, K., et al. Exon skipping and dystrophin restoration in patients with Duchenne muscular dystrophy after systemic phosphorodiamidate morpholino oligomer treatment: An open-label, phase 2, dose-escalation study. Lancet. 2011; 378(9791): 595-605.

[20] Kinali, M., Arechavala-Gomeza, V., Feng, L., Cirak, S., Hunt, D., and Adkin, C., et al. Local restoration of dystrophin expression with the morpholino oligomer AVI-4658 in Duchenne muscular dystrophy: A single-blind, placebocontrolled, dose-escalation, proof-of-concept study. Lancet Neurol. 2009; 8(10): 918-928.

[21] Goemans, N. M., Tulinius, M., van den Akker, J. T., Burm, B. E., and Ekhart, P. F., Heuvelmans, N., et al. Systemic administration of PRO051 in Duchenne's muscular dystrophy. N Engl J Med. 2011; 364(16): 1513-1522.

[22] Van Deutekom, J. C., Janson, A. A., Ginjaar, I. B., Frankhuizen, W. S., Aartsma-Rus, A., and Bremmer-Bout, M., et al. Local dystrophin restoration with antisense oligonucleotide PRO051. N Engl J Med. 2007; 357(26): 2677-2686.

[23] Aartsma-Rus, A. Overview on DMD exon skipping. Methods Mol Biol. 2012; 867: 97-116.

[24] Bowles, D. E., McPhee, S. W., Li, C., Gray, S. J., Samulski, J. J., and Camp, A. S., et al. Phase 1 gene therapy for Duchenne muscular dystrophy using a translational optimized AAV vector. Mol Ther. 2012; 20(2): 443-455.

[25] Van Deutekom, J. C., and Van Ommen, G. J. Advances in Duchenne muscular dystrophy gene therapy. Nat Rev Genet. 2003; 4(10): 774-783.

[26] Skuk, D., Goulet, M., Roy, B., Chapdelaine, P., Bouchard, J. P., and Roy, R., et al. Dystrophin expression in muscles of duchenne muscular dystrophy patients after high-density injections of normal myogenic cells. J Neuropathol Exp Neurol. 2006; 65(4): 371-386.

[27] Tsigkos, S., Llinares, J., Mariz, S., Aarum, S., Fregonese, L., and Dembowska-Baginska, B., et al. Use of biomarkers in the context of orphan medicines designation in the European Union. Orphanet J Rare Dis. 2014; 9(1): 13.

[28] Arechavala-Gomeza, V., Kinali, M., Feng, L., Guglieri, M., Edge, G., and Main, M., et al. Revertant fibres and dystrophin traces in Duchenne muscular dystrophy: Implication for clinical trials. Neuromuscul Disord. 2010; 20(5): 295-301.

[29] Arechavala-Gomeza, V., Kinali, M., Feng, L., Brown, S. C., Sewry, C., and Morgan. J. E., et al. Immunohistological intensity measurements as a tool to assess sarcolemma-associated protein expression. Neuropathol Appl Neurobiol. 2010;36(4): 265-274.

[30] Taylor, L. E., Kaminoh, Y. J., Rodesch, C. K., and Flanigan, K. M. Quantification of dystrophin immunofluorescence in dystrophinopathy muscle specimens. Neuropathol Appl Neurobiol. 2012; 38(6): 591-601.

[31] Anderson, L. V., and Davison, K. Multiplex Western blotting system for the analysis of muscular dystrophy proteins. Am J Pathol. 1999; 154(4): 1017-1022.

[32] Ishikawa, Y., Ishikawa, Y., and Minami, R. Quantitative estimation of dystrophin protein: A sensitive and convenient "two-antibody sandwich" ELISA. Tohoku J Exp Med. 1996; 180(1): 57-63.

[33] Brown, K. J., Marathi, R., Fiorillo, A. A., Ciccimaro, E. F., Sharma, S., and Rowlands, D. S., et al. Accurate Quantitation of Dystrophin Protein in Human Skeletal Muscle Using Mass Spectrometry. J Bioanal Biomed. 2012; Suppl 7.

[34] Escher, C., Lochmuller, H., Fischer, D., Frank, S., Reimann, J., and Walter, M. C., et al. Reverse protein arrays as novel approach for protein quantification in muscular dystrophies. Neuromuscul Disord. 2010; 20(5): 302-309.

[35] Cirak, S., Feng, L., Anthony, K., Arechavala-Gomeza, V., Torelli, S., and Sewry, C., et al. Restoration of the dystrophinassociated glycoprotein complex after exon skipping therapy in Duchenne muscular dystrophy. Mol Ther. 2012; 20(2): 462-467.

[36] Beekman, C., Testerink, J., Giannakopoulos, S., Kreuger, D., Sipkens, J., and Van Deutekom, J. C., et al. An objective method for immunofluorescence analysis of dystrophin levels in muscle from DMD patients in clinical studies. Neuromuscul Disord. 2013; 23(9-10): 812. Meeting report

[37] Lourbakos, A., beekman, C., Holling, T., Testerink, J., Duinsbergen, D., and Giannakopoulos, S., et al. Bioanalysis of a double blind, placebo-controlled clinical phase 2 study of drisapersen for the treatment of boys suffering from Duchenne muscular dystrophy and comparison to clinical outcome results. Neuromuscul Disord. 2013; 23(9-10): 805. Meeting report

[38] Aartsma-Rus, A., Janson, A. A., Kaman, W. E, BremmerBout, M., Den Dunnen, J. T., and Baas, F., et al. Therapeutic antisense-induced exon skipping in cultured muscle cells from six different DMD patients. Hum Mol Genet. 2003; 12(8): 907-914.

[39] Muntoni, F. The development of antisense oligonucleotide therapies for Duchenne muscular dystrophy: Report on a TREAT-NMD workshop hosted by the European Medicines Agency (EMA), on September 25th 2009. Neuromuscul Disord. 2010; 20(5): 355-362.

[40] Aartsma-Rus, A., and Muntoni, F. 194th ENMC international workshop. 3rd ENMC workshop on exon skipping: Towards clinical application of antisense-mediated exon skipping for Duchenne muscular dystrophy 8-10 December 2012, Naarden, The Netherlands. Neuromuscul Disord. 2013; 23(11): 934-944.

[41] Spitali, P., van den Bergen, J. C., Verhaart, I. E., Wokke, B., Janson, A. A., and van den Eijnde, R., et al. DMD transcript imbalance determines dystrophin levels. FASEB J. 2013; 27(12): 4909-4916.

[42] Veltrop, M., van der Kaa, J., Claassens, J., van, V. L., Verbeek, S., and Aartsma-Rus, A. Generation of embryonic stem cells and mice for duchenne research. PLoS Curr. 2013; 5.

[43] Wokke, B. H., van den Bergen, J. C., Versluis, M. J., Niks, E. H., Milles, J., and Webb, A. G., et al. Quantitative MRI and strength measurements in the assessment of muscle quality in Duchenne muscular dystrophy. Neuromuscul Disord. 2014.

[44] Henricson, E., Abresch, R., Han, J. J., Nicorici, A., Goude, K. E., and de, B. E., et al. The 6-minute walk test and personreported outcomes in boys with duchenne muscular dystrophy and typically developing controls: Longitudinal comparisons 
and clinically-meaningful changes over one year. PLoS Curr. 2013; 5 .

[45] Pane, M., Mazzone, E. S., Sormani, M. P., Messina, S., Vita, G. L., and Fanelli, L., et al. 6 minute walk test in Duchenne MD patients with different mutations: 12 month changes. PLoS One. 2014; 9(1): e83400.

[46] Goemans, N., van den Hauwe, M., Wilson, R., van, I. A., Klingels, K., and Buyse, G. Ambulatory capacity and disease progression as measured by the 6-minute-walk-distance in Duchenne muscular dystrophy subjects on daily corticosteroids. Neuromuscul Disord. 2013; 23(8): 618-623.

[47] McDonald, C. M., Henricson, E. K., Abresch, R. T., Florence, J. M, Eagle, M., and Gappmaier, E., et al. The 6-minute walk test and other endpoints in Duchenne muscular dystrophy: Longitudinal natural history observations over 48 weeks from a multicenter study. Muscle Nerve. 2013; 48(3): 343-356.

[48] Hoffman, E. P., Fischbeck, K. H., Brown, R. H., Johnson, M., Medori, R., and Loike, J.D., et al. Characterization of dystrophin in muscle-biopsy specimens from patients with Duchenne's or Becker's muscular dystrophy. N Engl J Med. 1988; 318(21): 1363-1368.

[49] van Putten, M., Hulsker, M., Young, C., Nadarajah, V. D., Heemskerk, H., and van der Weerd, L., et al. Low dystrophin levels increase survival and improve muscle pathology and function in dystrophin/utrophin double-knockout mice. FASEB J. 2013; 27(6): 2484-2495.

[50] Li, D., Yue, Y., and Duan, D. Marginal level dystrophin expression improves clinical outcome in a strain of dystrophin/utrophin double knockout mice. PLoS One. 2010; 5(12): e15286.

[51] Mirza, A., Sagathevan, M., Sahni, N., Choi, L., and Menhart, N. A biophysical map of the dystrophin rod. Biochim Biophys Acta. 2010; 1804(9): 1796-1809.

[52] Le, R. E., Hubert, J. F., Winder, S. J. A new twist to coiled coil. FEBS Lett. 2012; 586(17): 2717-2722.

[53] Flanigan, K. M., Voit, T., Rosales, X. Q., Servais, L., Kraus, J. E., Wardell, C., et al. Pharmacokinetics and safety of single doses of drisapersen in non-ambulant subjects with Duchenne muscular dystrophy: Results of a double-blind randomized clinical trial. Neuromuscul Disord. 2014; 24(1): 16-24.

[54] Hirawat, S., Welch, E. M., Elfring, G. L., Northcutt, V. J., Paushkin, S., and Hwang, S., et al. Safety, tolerability, and pharmacokinetics of PTC124, a nonaminoglycoside nonsense mutation suppressor, following single- and multiple-dose administration to healthy male and female adult volunteers. J Clin Pharmacol. 2007; 47(4): 430-444. 